\title{
The Garlic Preparation as an Alternative Way for Gastroprotection: From Bench to Clinic
}

\author{
Jong-Jae Park \\ Division of Gastroenterology, Department of Internal Medicine, Korea University Guro Hospital, Korea University College of Medicine, Seoul, \\ Korea
}

See "Gastroprotective Effects of PMK-S005 against Ethanol-Induced Acute Gastric Damage in Rats" by Yoon Jeong Choi, et al. on page 348-355, Vol. 10. No. 3, 2016

Health benefit of garlic has been well established for a longtime during the history of mankind. Organosulfur compounds of garlic including $\delta$-glutamyl-s-allyl-L-cysteins and $s^{-}$-allyl- ${ }_{-}-$ cysteine (SAC) sulfoxides are known as major components of bioactivity; however, raw garlic is easy to be converted into bio-inactive form. Thus, many types of garlic extract or preparation with diverse composition of bioactive compounds have been developed and their bio-efficacy has been evaluated in experimental research. Commercially available garlic preparations including aged garlic extract, essential oil, macerates and powder have been reported to have health-promoting and beneficial effect. ${ }^{1}$ In concrete, garlic extract has been reported to show antioxidant effect by scavenging reactive oxygen species (ROS) and induction of antioxidant enzymes, inhibit lipoprotein oxidation, lower serum glucose and have antitumorigenic effect by promoting apoptosis and induction of cell cycle arrest. ${ }^{2}$ Garlic extract has been also studied to show suppressive effect of Helicobacter pylori-induced gastric inflammation in vivo ${ }^{3}$ and reduction of gastric cancer incidence in a clinical trial. ${ }^{4}$ Among them, SAC is one of a water-soluble organosulfur compound of aged garlic extract, and its powerful protective effects in various medical fields have been spotlighted nowadays, and gastrointestinal tract is no exception. In this study, Choi et al. ${ }^{5}$ are focusing on the gastroprotective effect of a synthetic SAC in vivo by using ethanol-induced acute gastric ulcer model in rats.

The authors of this article have performed experimental animal studies for discovery of novel natural compound, such as Cochinchina momordica seed extract, for defensive effect from gastric mucosal injury using ethanol or stress-induced gastric mucosal damage model in rats. ${ }^{6}$ They also have researched on establishment of adequate rat models for acute gastric inflammation, ${ }^{7}$ and the impact of aging on gastric mucosal injury by altering defense mechanism of apoptosis, angiogenesis and sensory neuron activity. ${ }^{8}$ Through consistent investigation, the authors showed that various factors associated with mechanism of gastric mucosal damage, such as proinflammatory cytokines, gastric mucins, ROS, can be modified by aging or alternative natural compounds, and consequently mucosal injury can be enhanced or suppressed. Their consistent works toward understanding and complete conquest of gastric mucosal damage are really worthy of compliments.

This study is basically an extension of their previous study, which demonstrated the protective effects of PMK-S005, a synthetic SAC, using nonsteroidal anti-inflammatory drugs (NSAID)-induced acute gastric damage in rats. In the previous study, authors established NSAID-induced gastric mucosal injury by using 8-week Sprague-Dawley rats with administration of aspirin, diclofenac and indomethacine. PMK-S005 (1, 5, or $10 \mathrm{mg} / \mathrm{kg}$ ) or rebamipide, an established gastroprotective drug by producing prostaglandin, were pretreated and their pharmacologic effects were evaluated by measuring gross ulcer index, histologic index, gastric mucus level, mucosal level of inflammatory markers including myeloperoxidase, tumor-necrosis factor $\alpha$, interleukin $1 \beta$ and several molecules associated with prostaglandin-synthesis pathway including prostaglandin E2 $\left(\mathrm{PGE}_{2}\right)$ and leukotriene $\mathrm{B} 4\left(\mathrm{LTB}_{4}\right)^{9}{ }^{9}$

A portion of basic experimental design of the present study is derived from authors' previous work above mentioned,

Correspondence to: Jong-Jae Park

Division of Gastroenterology, Department of Internal Medicine, Korea University Guro Hospital, Korea University College of Medicine, 148 Gurodong-ro, Guro-gu, Seoul 08308, Korea

Tel: +82-2-2626-1027, Fax: +82-2-2626-1038, E-mail: gi7pij@korea.ac.kr pISSN 1976-2283 eISSN 2005-1212 http://dx.doi.org/10.5009/gnl16138

(a) This is an Open Access article distributed under the terms of the Creative Commons Attribution Non-Commercial License (http://creativecommons.org/licenses/by-nc/4.0) which permits unrestricted non-commercial use, distribution, and reproduction in any medium, provided the original work is properly cited. 
except that acute gastric ulcers were induced by administration of ethanol instead of NSAIDs. However, authors further analyzed the change of antioxidant enzyme including heme oxygenase-1 (HO-1), NAD(P)H:quinine oxidoreductase 1 (NQO1), $\gamma$-glutamylcysteine catalytic subunit (GCLC) and modulator subunit (GCLM). The effects of PMK-S005 for gastric ulcer healing, improvement of histopathologic parameters and mucosal level of pro-inflammatory markers and $\mathrm{LTB}_{4}$, a prostaglandin synthesis-associated factor, were consistent with those of the previous study. However, authors additional revealed in this study that the long-term treatment of PMK-S005 (5 or $10 \mathrm{mg} /$ $\mathrm{kg}$ ) for 14 days significantly increased protein expression level of HO-1, NQO-1, GCLC and GCLM in a dose-dependent manner, which suggest that gastroprotective effects of PMK-S005 can be caused by enhancement of antioxidant enzymes. ${ }^{5}$ Data from another previous research by Park et al. ${ }^{10}$ also support the results of this study, which showed that anti-inflammatory and mucosal protective effects of SAC in indomethacin-induced acute gastric ulcer model in rats were mediated by inhibition of cyclooxygenase- 2 signaling and histone deacetylation activity, and induction of antioxidant including HO- $1 .{ }^{10}$ An interesting thing is that PMK-S005 significantly suppressed the mucosal level of $\mathrm{LTB}_{4}$, but did not alter $\mathrm{PGE}_{2}$. Furthermore, mucosal PGE 2 level was not significantly altered by ethanol ingestion at first, which suggest that a different molecular mechanism may be involved in ethanol-induced gastric ulcer model, comparing with NSAIDinduced gastric ulcer.

Two principal risk factors of gastric ulcers or mucosal injury are $H$. pylori infection and long-term use of NSAIDs, and the mainstay of treatment of gastric ulcer is eradication of $H$. pylori and inhibition of acid secretion by using $\mathrm{H}_{2}$-receptor antagonist or proton pump inhibitors. However, main mechanism of development of gastric ulcer and recurrence is imbalance between gastric mucosal defensive factors including prostaglandin, bicarbonate and mucus and offensive factors including acid and pepsin. Thus, gastroprotective drugs such as rebamipide or sucralfate, which can efficiently enhance mucosal defensive factors such as prostaglandin, have been used worldwide. Furthermore, development and evaluation of efficacy of natural product, such as Artemisia, Pongamia pinnata, Lafoensia pacari, for gastroprotection have been being vigorously performed, and these alternative drugs may increase the quality of ulcer healing by improving mucosal defense and lower the likelihood of recurrence. ${ }^{11}$ In this regards, the aim of this article to evaluate the gastroprotective efficacy of synthetic SAC and investigating underlying mechanism is well-timely, and presents a potential for alternative and effective drug for gastroprotection. In real world, however, no one could have answered the simple question: Is it true that Asian people, especially Korean and Chinese, have any benefit from garlic-rich food culture in terms of gastric ulcer? It will take a long way until synthetic SAC is developed as a commercially available drug and evaluated in the clinical fields in the future. We believe that such efforts will eventually lead us to step closer toward the complete conquest of treatment of benign gastric ulcer.

\section{CONFLICTS OF INTEREST}

No potential conflict of interest relevant to this article was reported.

\section{REFERENCES}

1. Butt MS, Sultan MT, Butt MS, Iqbal J. Garlic: nature's protection against physiological threats. Crit Rev Food Sci Nutr 2009;49:538551.

2. Trio PZ, You S, He X, He J, Sakao K, Hou DX. Chemopreventive functions and molecular mechanisms of garlic organosulfur compounds. Food Funct 2014;5:833-844.

3. Iimuro M, Shibata H, Kawamori T, et al. Suppressive effects of garlic extract on Helicobacter pylori-induced gastritis in Mongolian gerbils. Cancer Lett 2002;187:61-68.

4. Ma JL, Zhang L, Brown LM, et al. Fifteen-year effects of Helicobacter pylori, garlic, and vitamin treatments on gastric cancer incidence and mortality. J Natl Cancer Inst 2012;104:488-492.

5. Choi YJ, Kim N, Lee JY, et al. Gastroprotective effects of PMKS005 against ethanol-induced acute gastric damage in rats. Gut Liver 2016;10:348-355.

6. Kang JM, Kim N, Kim B, et al. Gastroprotective action of Cochinchina momordica seed extract is mediated by activation of CGRP and inhibition of cPLA(2)/5-LOX pathway. Dig Dis Sci 2009;54: 2549-2560.

7. Lee BH, Kim N, Nam RH, et al. Difficult establishment of a chronic nonsteroidal anti-inflammatory drugs induced gastric inflammation rat model due to gastric adaptation and small bowel damage. Korean J Gastroenterol 2014;63:341-347.

8. Kang JM, Kim N, Kim JH, et al. Effect of aging on gastric mucosal defense mechanisms: ROS, apoptosis, angiogenesis, and sensory neurons. Am J Physiol Gastrointest Liver Physiol 2010;299:G1147G1153.

9. Choi YJ, Kim N, Lee JY, et al. Protective effects of garlic extract, PMK-S005, against nonsteroidal anti-inflammatory drugs-induced acute gastric damage in rats. Dig Dis Sci 2014;59:2927-2934.

10. Park JM, Han YM, Kangwan N, et al. S-allyl cysteine alleviates nonsteroidal anti-inflammatory drug-induced gastric mucosal damages by increasing cyclooxygenase-2 inhibition, heme oxygenase-1 induction, and histone deacetylation inhibition. J Gastroenterol Hepatol 2014;29 Suppl 4:80-92.

11. Kangwan N, Park JM, Kim EH, Hahm KB. Quality of healing of gastric ulcers: natural products beyond acid suppression. World J Gastrointest Pathophysiol 2014;5:40-47. 